\title{
Multiparametric whole-body 3.0-T MRI in newly diagnosed intermediate- and high-risk prostate cancer: diagnostic accuracy and interobserver agreement for nodal and metastatic staging
}

\author{
Edward William Johnston ${ }^{1} \cdot$ Arash Latifoltojar $^{1} \cdot$ Harbir Singh Sidhu ${ }^{1} \cdot$ Navin Ramachandran ${ }^{1} \cdot$ Magdalena Sokolska $^{2}$. \\ Alan Bainbridge ${ }^{2} \cdot$ Caroline Moore $^{3} \cdot$ Hashim Uddin Ahmed ${ }^{4} \cdot$ Shonit Punwani $^{1}$ (D)
}

Received: 12 April 2018 / Revised: 24 August 2018 / Accepted: 28 September 2018 / Published online: 5 December 2018

(C) The Author(s) 2018

\begin{abstract}
Objectives To determine the diagnostic accuracy and interobserver concordance of whole-body (WB)-MRI, vs. ${ }^{99 \mathrm{~m}} \mathrm{Tc}$ bone scintigraphy (BS) and ${ }^{18}$ fluoro-ethyl-choline $\left({ }^{18} \mathrm{~F}\right.$-choline) $\mathrm{PET} / \mathrm{CT}$ for the primary staging of intermediate/high-risk prostate cancer.

Methods An institutional review board approved prospective cohort study carried out between July 2012 and November 2015, whereby 56 men prospectively underwent 3.0-T multiparametric (mp)-WB-MRI in addition to BS (all patients) $\pm{ }^{18} \mathrm{~F}$-choline PET/CT (33 patients). MRI comprised pre- and post-contrast modified Dixon (mDixon), T2-weighted (T2W) imaging, and diffusion-weighted imaging (DWI). Patients underwent follow-up mp-WB-MRI at 1 year to derive the reference standard. WBMRIs were reviewed by two radiologists applying a 6-point scale and a locked sequential read (LSR) paradigm for the suspicion of nodal $(\mathrm{N})$ and metastatic disease (M1a and M1b).

Results The mean sensitivity/specificity of WB-MRI for N1 disease was 1.00/0.96 respectively, compared with 1.00/0.82 for ${ }^{18} \mathrm{~F}$-choline PET/CT. The mean sensitivity and specificity of WB-MRI, ${ }^{18} \mathrm{~F}$-choline PET/CT, and BS were $0.90 / 0.88,0.80 / 0.92$, and $0.60 / 1.00$ for M1b disease. ROC-AUC did not show statistically significant improvement for each component of the LSR; mean ROC-AUC 0.92, 0.94, and $0.93(p<0.05)$ for mDixon + DWI, + T2WI, and + contrast respectively. WB-MRI had an interobserver concordance $(\kappa)$ of $0.79,0.68$, and 0.58 for $\mathrm{N} 1, \mathrm{M} 1 \mathrm{a}$, and M1b diseases respectively.

Conclusions WB-MRI provides high levels of diagnostic accuracy for both nodal and metastatic bone disease, with higher levels of sensitivity than BS for metastatic disease, and similar performance to ${ }^{18} \mathrm{~F}$-choline PET/CT. T2 and post-contrast mDixon had no significant additive value above a protocol comprising mDixon and DWI alone.

Key Points

- A whole-body MRI protocol comprising unenhanced mDixon and diffusion-weighted imaging provides high levels of diagnostic accuracy for the primary staging of intermediate- and high-risk prostate cancer.

- The diagnostic accuracy of whole-body MRI is much higher than that of bone scintigraphy, as currently recommended for clinical use.

- Staging using WB-MRI, rather than bone scintigraphy, could result in better patient stratification and treatment delivery than is currently provided to patients worldwide.
\end{abstract}

Electronic supplementary material The online version of this article (https://doi.org/10.1007/s00330-018-5813-4) contains supplementary material, which is available to authorized users.

Shonit Punwani

shonit.punwani@gmail.com

1 UCL Centre for Medical Imaging, 2nd Floor Charles Bell House, 43 45 Foley Street, London W1W 7TS, UK

2 Medical Physics, University College London Hospital, 235 Euston Road, London NW1 2BU, UK
3 Department of Urology, University College Hospital, 235 Euston Road, London NW1 2BU, UK

4 Department of Urology, Imperial College London, Fulham Palace Road, Hammersmith, London W6 8RF, UK 
Keywords Magnetic resonance imaging $\cdot$ Prostate $\cdot$ Choline $\cdot$ Positron emission tomography

$\begin{array}{ll}\text { Abbreviations } & \\ \text { BS } & \text { Bone scintigraphy } \\ \text { BVC } & \text { Best value comparator } \\ \text { DWI } & \text { Diffusion-weighted imaging } \\ \text { LSR } & \text { Locked sequential read } \\ \text { mp-WB-MRI } & \text { Multiparametric whole-body MRI } \\ \text { MTB } & \text { Multidisciplinary tumour board } \\ \text { PLND } & \text { Pelvic lymph node dissection } \\ \text { PSMA } & \text { Prostate-specific membrane antigen } \\ \text { ROC-AUC } & \text { Receiver operator characteristic area under } \\ & \text { the curve } \\ \text { SAD } & \text { Short-axis diameter }\end{array}$

\section{Introduction}

Since patient survival in intermediate- and high-risk prostate cancer depends heavily on TNM stage [1], accurate tumour staging should underpin all prognostication and management decisions. However, the mainstay of imaging-based staging decisions is still based on ${ }^{99 \mathrm{~m}} \mathrm{Tc}$ bone scintigraphy (BS) \pm pelvic $\mathrm{CT}$, as is still advised at least eight international guidelines [2]. Whilst these modalities are simple to implement, their diagnostic accuracy remains severely limited [3, 4], which has driven the development of a number of imaging methods for cancer staging,

While choline PET/CT offers improved sensitivity and specificity for both nodal $[4,5]$ and metastatic disease vs. BS and conventional CT [4], PET involves ionising radiation exposure and has a spatial resolution limited to $5 \mathrm{~mm}$ [6], poor contrast resolution, and financial and logistical difficulties which limit its use. Whole-body (WB)-MRI offers potential solutions to these problems, with improved spatial and contrast resolution [7], lack of ionising radiation, and comparable performance characteristics to choline PET/CT as shown by a number of early studies [8-12]. Although prostate-specific membrane antigen (PSMA) PET/CT has demonstrated considerable early promise [13], its availability is limited and incurs considerable cost. Furthermore, since prostate cancer patients commonly undergo multiparametric $(\mathrm{mp})$ prostate MRI, the possibility of a one-stop staging modality has been raised [12] whereby mp-WB approaches could also be applied. However, the interobserver concordance of WB-MRI remains uncertain, as does a definition of what constitutes an optimal acquisition. Further validation regarding diagnostic accuracy is also required.

The primary aim of the present study is to determine the diagnostic accuracy of WB-MRI vs. BS and ${ }^{18}$ fluoro-ethyl-choline $\left({ }^{18} \mathrm{~F}\right.$-choline) PET/CT for the primary staging of intermediateand high-risk prostate cancer, using a multiparametric vertex-to- feet acquisition protocol and a locked sequential read (LSR) paradigm to determine the additive value of each MRI sequence. Secondary aims include assessment of lesion distribution, interobserver concordance, and intermodality concordance with BS and ${ }^{18} \mathrm{~F}$-choline PET/CT. We hypothesise that (i) WB-MRI has a higher diagnostic accuracy than BS, (ii) WB-MRI has good interobserver concordance, and (iii) a multiparametric whole-body acquisition has a greater diagnostic accuracy than T1-weighted imaging plus DWI.

\section{Materials and methods}

Our institutional review board approved this prospective single-centre study. Informed written consent was obtained from each participant, whereby 56 consecutive men (mean age 67.9 years, range 51.9-84.4) were identified at Multidisciplinary Tumour Board (MTB) meetings and recruited to the study between July 2012 and November 2015 . Inclusion criteria were (i) men aged 18 or over and (ii) new diagnosis of intermediate- or high-risk prostate cancer according to the D'Amico criteria [14]. Exclusion criteria were (i) contraindications to MRI, e.g. severe claustrophobia or MR unsafe device, (ii) prior therapy for prostate cancer, and (iii) men unable to provide informed consent. A recruitment flow diagram is shown in Fig. 1.

Standard imaging comprised BS in all patients $\pm{ }^{18} \mathrm{~F}$-choline $\mathrm{PET} / \mathrm{CT}$, in 33 patients. The decision to perform a ${ }^{18} \mathrm{~F}$-choline PET/CT was made on a case-by-case basis whereby the risk of extraprostatic disease was considered to be high at MTB discussion; however, the result of the WB-MRI was blinded to the MTB members, so it did not influence the decision to perform PET/CT. WB-MRI was performed within a mean of 15.9 days (range $0-49$ ) of BS.

\section{Multiparametric WB-MRI protocol}

All patients were imaged on a 3.0-T wide-bore system (Ingenia, Philips), with whole-body coverage from the vertex to feet using a head coil, two anterior surface coils, and tableembedded posterior coils. Coronal pre-contrast modified Dixon (mDixon), axial T2 turbo spin echo (TSE), and axial diffusion-weighted imaging (DWI) with body signal suppression at $4 b$-values (b0, b100, b300, and b1000) were performed, from which an ADC map was constructed. Postcontrast mDixon imaging was then carried out following a $20 \mathrm{ml}$ injection of intravenous gadoterate meglumine (Dotarem ${ }^{\circledR}$, Guebert).

Full acquisition parameters are provided in Table 1. 


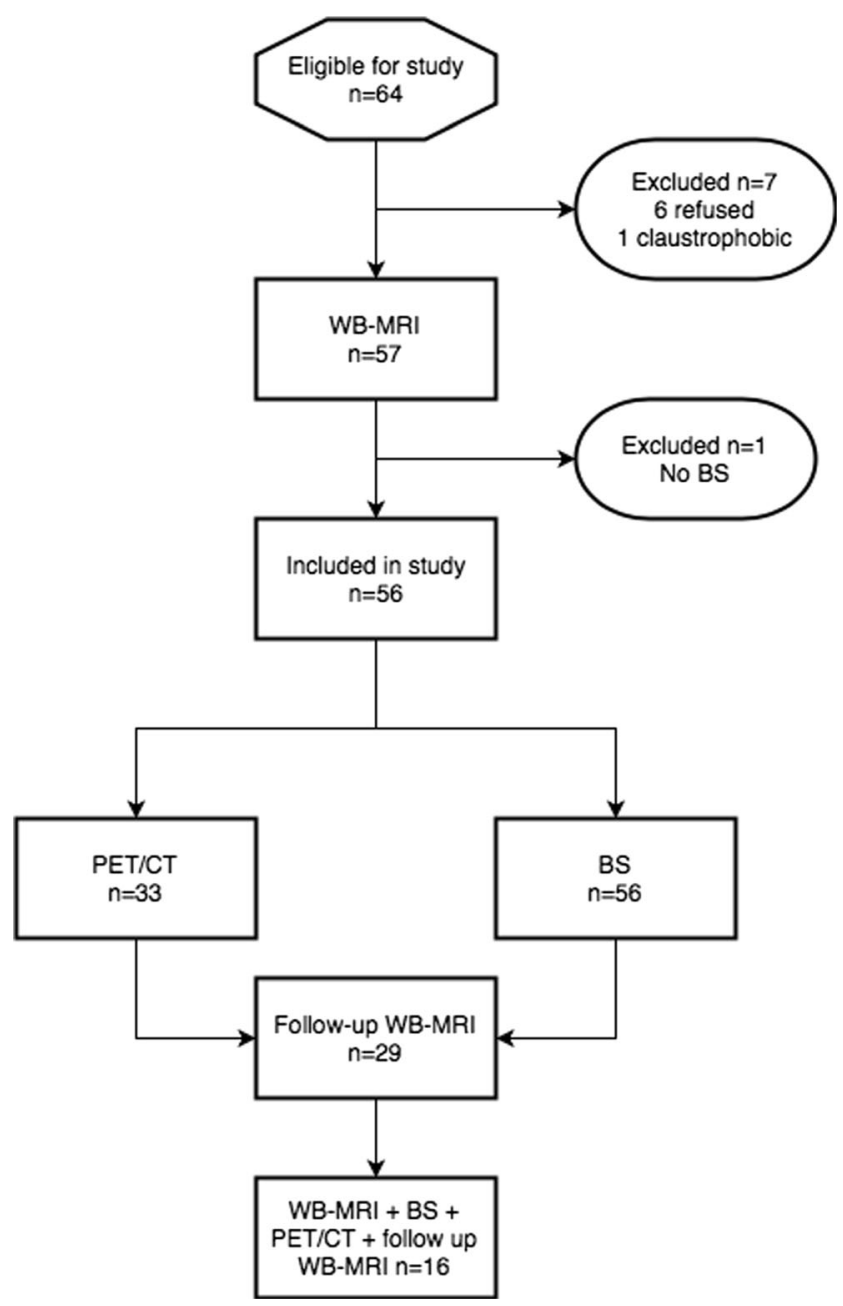

Fig. 1 Patient recruitment flow diagram

\section{${ }^{99 m}$ Tc scintigraphy protocol}

Whole-body imaging was performed in all patients, using anterior and posterior views, $256 \times 1024$ matrix, and energy window(s) of $140 \mathrm{keV}, 2-4 \mathrm{~h}$ after a single injection of $\mathrm{Tc}^{99} \mathrm{~m}$-methylene diphosphonate (MDP).

\section{${ }^{18}$ F-choline PET/CT protocol}

Thirty-three patients underwent ${ }^{18} \mathrm{~F}$-choline PET/CT on an integrated 64-slice scanner (Discovery VCT; GE Healthcare) from the vertex to mid-thigh, $60 \mathrm{~min}$ after an intravenous injection of ${ }^{18} \mathrm{~F}$-fluoro-ethyl-choline tracer (198-410 MBq; average activity, 327.4 MBq). A low-dose, unenhanced CT scan was initially performed for attenuation correction and image fusion at $120 \mathrm{keV}$ and $10 \mathrm{~mA}$ with couch movement $0.8 \mathrm{~s}$ and $30 \mathrm{~mm}$ per rotation. Whole-body PET emission images were then acquired and reconstructed using the Houndsfield units from the CT to a resolution of $128 \times 128$ with 5-mm slice thickness.

\section{Follow-up WB-MRI}

Patients were invited to attend a follow-up WB-MRI 1 year after their initial scan using an identical acquisition protocol to inform the reference standard. Twenty-nine of the fifty-six patients attended the 1-year scan, and 16 of these patients had undergone PET-CT at baseline (Fig. 1). Of the 27 who did not attend, two patients died, 16 refused a second attendance, and 9 were lost to follow-up.

\section{BS and ${ }^{18} \mathrm{~F}$-choline PET/CT image review}

Nuclear medicine physicians reviewed the BS and ${ }^{18} \mathrm{~F}$ choline PET/CT staging studies as part of standard clinical care using GE Advantage workstations. Disease positivity was defined as accumulation of radiotracer, greater than the surrounding background and incompatible with normal physiological activity.

\section{WB-MRI review}

Images were prepared for review using the scanner workstation for mDixon images and Osirix (v. 7.0 Pixmeo). Two board-certified radiologists (reader 1, NR with 12 years of experience and reader 2, HS with 9 years of experience) independently reviewed anonymised WB-MR datasets using an Osirix workstation (v. 7.0 Pixmeo), aware of the presenting serum prostate-specific antigen (PSA) level only and blinded to all other clinical and imaging results.

The body was divided into nine nodal regions (external iliac, internal iliac, common iliac, paraaortic, presacral, other abdominal, inguinal, thoracic, and neck) using standard anatomic definitions. Ten skeletal sites were assessed for the presence of disease (skull, cervical spine, thoracic spine, lumbar spine, pelvis, sternum, clavicle/scapula, ribs, upper limb, and lower limb). Scans were reviewed using a LSR paradigm, whereby each radiologist initially reviewed the unenhanced mDixon and DWI and scored the suspicion of disease at each site using a 1-6 ordinal scale (1, definitely not present; 2 , probably not present; 3 , possibly not present; 4 , possibly present; 5 , probably present; 6 , definitely present) for each disease site, according to the TNM 7th edition staging system (N0/N1, M1a/M1b/M1c).

The score was specifically assigned at each site using the imaging features as follows on the pre-contrast mDixon and DWI sequences: 1 , no lesion evident; 2 , poorly visible lesion evident on T1-weighted imaging only-low T1 signal bone focus or lymph node visible but not convincing for malignant involvement or $<5$-mm short axis diameter (SAD); 3 , definite lesion visible on T1-weighted imaging but not DWI, lymph node 6-9 $\mathrm{mm}$ in SAD; 4, definite lesion on T1 with mild increase in high $b$-value diffusion signal vs. background noise, lymph node $10-12 \mathrm{~mm}$ in SAD; 5 , definite lesion seen on T1 
Table 1 Whole-body MRI acquisition parameters.

\begin{tabular}{lclc}
\hline Imaging plane & T2-TSE & $\begin{array}{l}\text { mDixon } \\
\text { pre- and post-contrast) } \\
\text { Coronal }\end{array}$ & DWI (b0, b100, b300, b1000) \\
& Transverse & Transverse \\
\hline TE $(\mathrm{ms})$ & 80 & $1.02 / 1.8$ & 71 \\
TR $(\mathrm{ms})$ & 1228 & 3.0 & 6371 \\
FOV $(\mathrm{mm} \times \mathrm{mm})$ & $500 \times 300$ & $502 \times 300$ & $500 \times 306$ \\
Voxel size $(\mathrm{mm} \times \mathrm{mm})$ & $1 \times 1$ & $2.1 \times 2.1$ & $4 \times 4.2$ \\
Number of slices & 40 & 120 & 40 \\
Slice thickness $(\mathrm{mm})$ & 5 & 5 & 5 \\
Acquisition matrix & $500 \times 286$ & $144 \times 238$ & $124 \times 72$ \\
ETL & 91 & 2 & 39 \\
Acceleration factor $(\mathrm{SENSE})$ & 2 & 2 & 2.5 \\
Pixel bandwidth $(\mathrm{Hz})$ & 537 & 1992 & 3369 \\
Scan time (min) & 15.2 & $5.5 \times 2$ & 47 \\
\hline
\end{tabular}

T2-TSE T2-weighted turbo spin echo, mDixon modified Dixon, DWI diffusion-weighted imaging, TE time of echo, TR time of repetition, FOV field of view, ETL echo train length, SENSE sensitivity encoding and DWI with moderate increase in high $b$-value DWI signal vs. background noise, lymph node 12-14 mm SAD; 6 , definite lesion seen on T1 and DWI with large increase in high $b$ value signal vs. background noise, lymph node $\geq 15 \mathrm{~mm}$ SAD. T2W images were then revealed and sites rescored as negative or positive. A negative score was assigned where there was no lesion or where features favoured benignity (e.g. fatty nodal hilum or high T2 signal of haemangioma), and a positive score assigned for features that favour malignancy (rounded nodal morphology, low T2 signal in node or bone lesion). Positive T2 appearances were scored up a point on the initial 1-6 scale (e.g. 3/6 on mDixon/DWI becomes $4 / 6$ ), and negative T2 appearances were scored down a point (3/6 on mDixon/DWI becomes 2/6). Lastly, post-contrast mDixon images were revealed and a final WB-MRI score was assigned. Here, lesional enhancement was scored up a point on the 1-6 scale and down a point if there was no enhancement. The time to report WB-MRI studies was recorded for both readers. Where discordancy arose between the two radiologists, a third board-certified radiologist with 12 years of experience (reader 3, SP) adjudicated and rescored discordant sites using all available MR images, also aware of the PSA level only.

\section{Derivation of WB-MRI reference standard}

A panel comprising two board-certified radiologists (SP and EJ with 12 and 6 years of experience), and an oncologist with 8 years of experience (RD) reviewed baseline and follow-up WB-MRIs, in combination with all available clinical and radiological information at least 1 year from baseline imaging to carry out a patient-based analysis, and assign patients into the following categories using the definitions below for all modalities. Patients were included in the M1a sensitivity/ specificity analysis if:

(i) they had undergone baseline WB-MRI and had a positive ${ }^{18} \mathrm{~F}$-choline PET/CT for nodal assessment; or

(ii) they had a baseline WB-MRI and negative ${ }^{18} \mathrm{~F}$-choline $\mathrm{PET} / \mathrm{CT}$ and also underwent follow-up WB-MRI to allow final arbitration.

Similarly, patients were included in the M1b sensitivity/ specificity analysis if:

(i) they had undergone baseline WB-MRI and had a positive ${ }^{18}$ F-choline PET/CT or BS for bone assessment; or

(ii) they had a baseline WB-MRI and negative ${ }^{18} \mathrm{~F}$-choline $\mathrm{PET} / \mathrm{CT}$ and BS, and also underwent follow-up WBMRI to allow final arbitration.

The reference standard was subsequently derived using the following definitions:

True positive (TP) sites:

(i) Lesion on WB-MRI (defined as suspicion level 4/5/6) which is $\mathrm{BS}$ and/or ${ }^{18} \mathrm{~F}$-choline PET/CT positive (if performed). Followup WB-MRI (if performed) also demonstrates lesion progression without systemic therapy, decrease with systemic therapy, or new lesions. (ii) Lesion on WB-MRI which is negative on conventional imaging but progresses on WB-MRI follow-up without systemic therapy, or new lesions appear on WB-MRI follow-up.

True negative (TN) sites: No lesion on WB-MRI (defined as suspicion level 1/2/3) and BS and ${ }^{18} \mathrm{~F}$-choline PET/CT concordantly negative. In addition, follow-up WB-MRI 
a

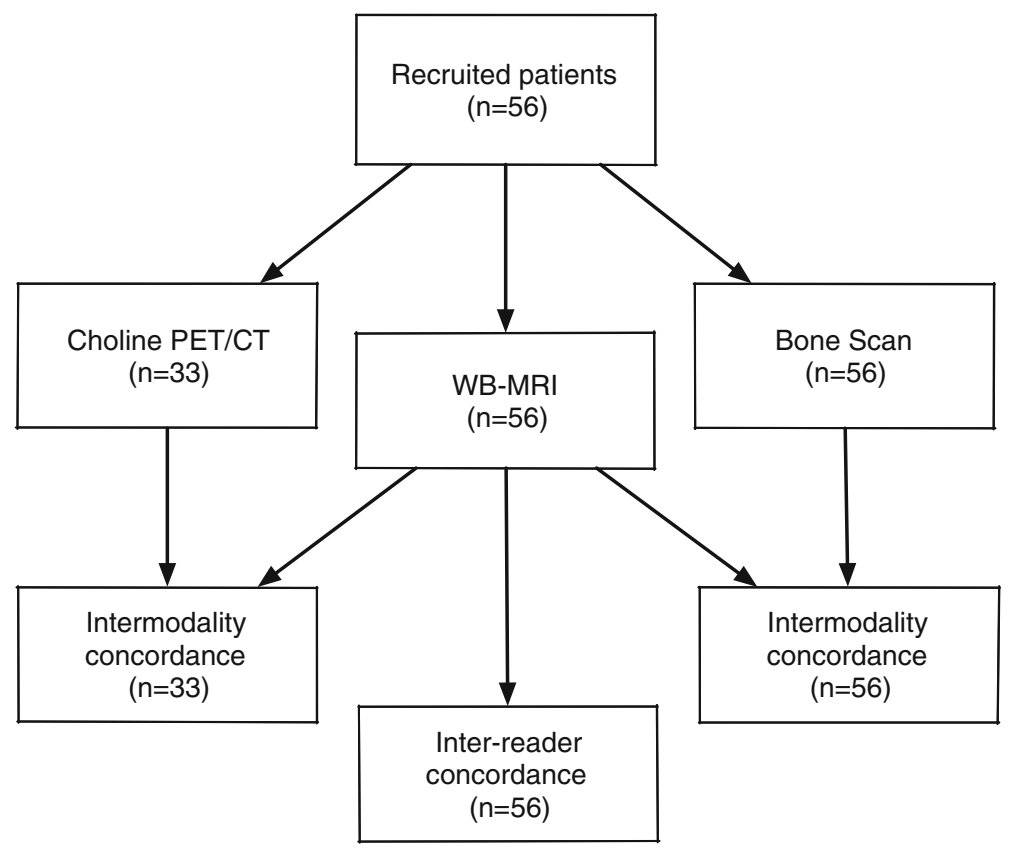

b

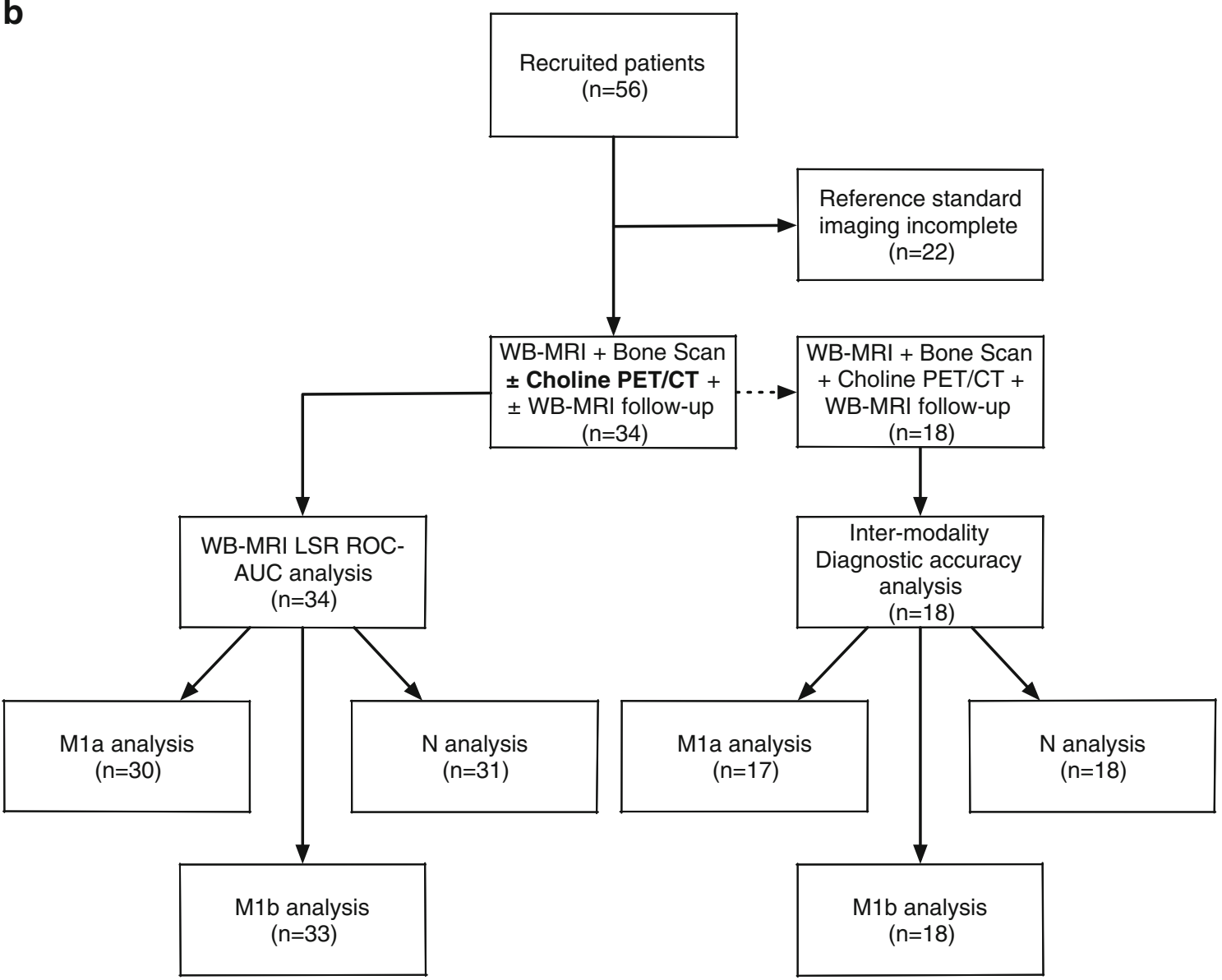

Fig. 2 Flow diagrams of the statistical methods used in the study. a Flow diagram of intermodality and inter-reader concordance. b Flow diagram for WB-MRI LSR ROC-AUC and intermodality diagnostic accuracy analyses. $\mathbf{c}$ Patient-based reference standard 
C

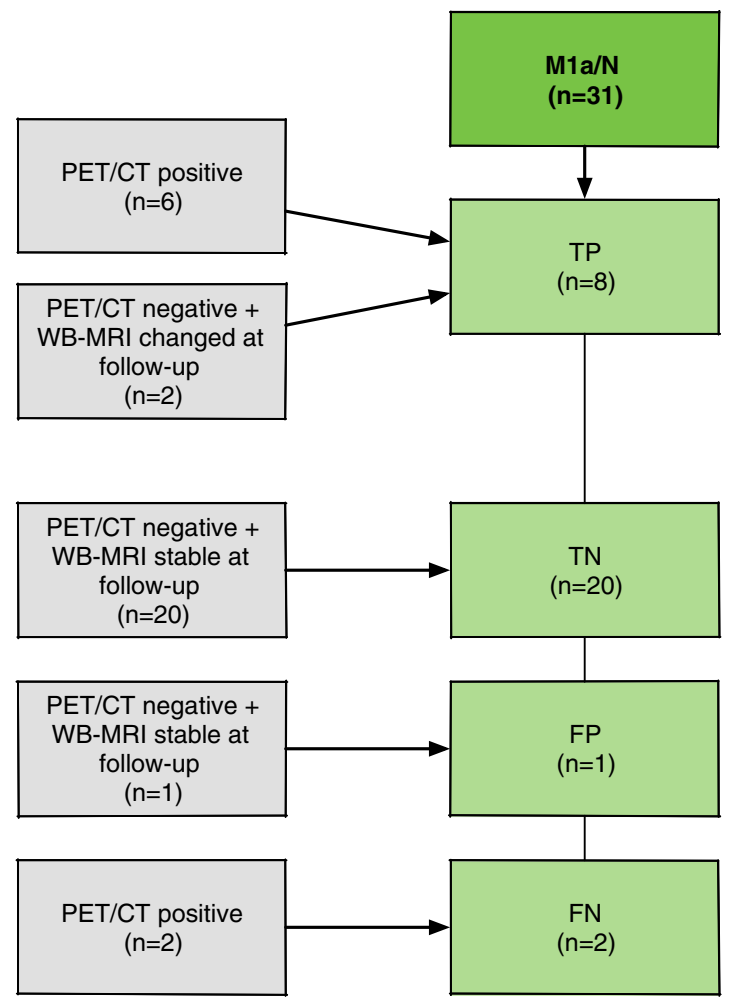

Fig. 2 (continued)

remains negative. WB-MRI follow-up was therefore required to assign $\mathrm{TN}$ cases.

False positive (FP) sites: Lesion on WB-MRI that was BS and ${ }^{18} \mathrm{~F}$-choline PET/CT negative and unchanged at follow-up. WB-MRI follow-up was therefore required to assign FP cases.

False negative (FN) sites: No lesion on WB-MRI but positive $\mathrm{BS}$ and/or ${ }^{18} \mathrm{~F}$-choline $\mathrm{PET} / \mathrm{CT}$.

A flow diagram of the statistical methods and reference standard used in this study is provided in Fig. 2.

A similar reference standard was used to compare BS and

${ }^{18} \mathrm{~F}$-choline PET/CT, whereby positive scans concordant with WB-MRI were considered as true positive, with follow-up WB-MRI used for arbitration of discordant and negative BS and ${ }^{18} \mathrm{~F}$-choline PET/CT findings. The details for this reference standard are provided in the supplementary materials.

\section{Statistical analysis}

Statistical analysis was performed using SPSS Statistics version 23 (2015, IBM) as below:

1. The distribution of positive lesions for each staging modality (BS, ${ }^{18} \mathrm{~F}$-choline PET/CT, and WB-MRI) for local nodal (N0/N1) and metastatic disease (M1a/M1b/M1c) using the

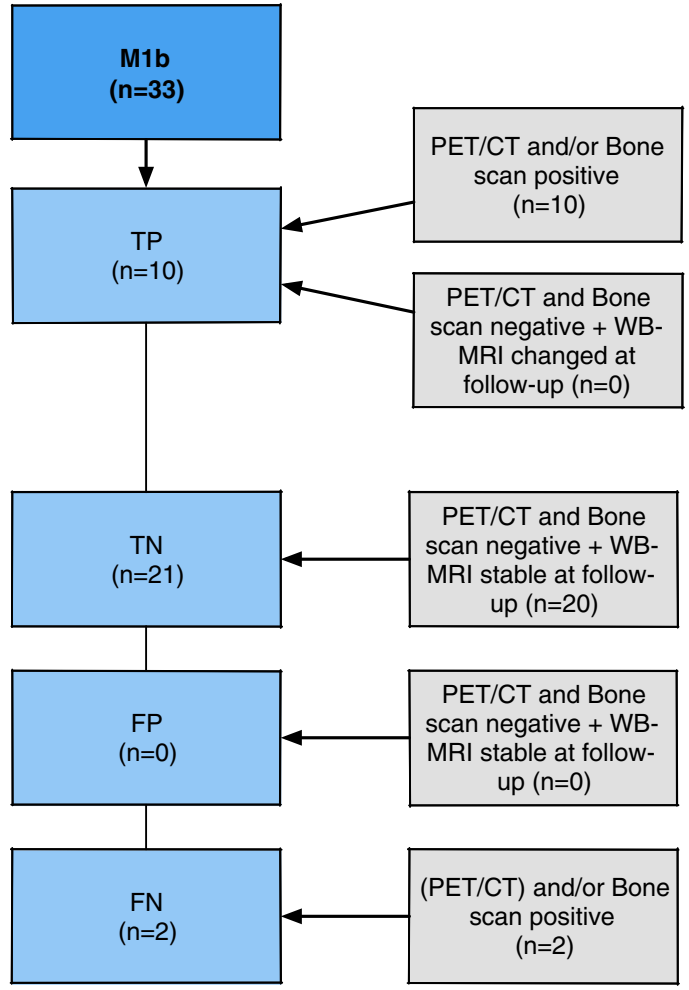

TNM classification, following the adjudication of discordant sites by the third board-certified radiologist. Percentages were recorded (i) for all patients $(n=56)$ and (ii) for patients undergoing ${ }^{18} \mathrm{~F}$-choline PET/CT ( $n=33$ ) (Fig. 2a).

2. The inter-reader agreement of WB-MRI $(n=56)$ and agreement between WB-MRI (following the consensus read) and BS $(n=56)$ and ${ }^{18} \mathrm{~F}$-choline PET/CT $(n=33)$ following adjudication by the third board-certified radiologist were assessed using Cohen's $\kappa$ statistics, interpreted according to Landis and Koch [15], whereby $<0$ indicates no agreement; $0-0.20$, slight; $0.21-0.40$, fair; $0.41-0.60$, moderate; $0.61-$ 0.80 , substantial; and $0.81-1$, almost perfect agreement (Fig. 2a).

3. Receiver operator characteristic area under the curve (ROCAUC) was calculated for WB-MRI studies (Fig. 2b), for both readers following each component of the LSR, applying thresholds for each level of suspicion (1-6) vs. the reference standard (Fig. 2c). Differences in ROC-AUC values for each component of the LSR were assessed according to [16], using a significance level of $p<0.05$. Youden's index [17] was used to determine the optimal cutoff of the ROC curve providing the highest combination of sensitivity and specificity.

4. An intermodality diagnostic accuracy study was performed (Fig. 2b), whereby the sensitivity, specificity positive (PPV), and negative (NPV) predictive values were then determined 
Table 2 Distribution of lesions on each imaging modality. The first row for each N/M stage represents a comparison between patients who underwent BS, ${ }^{18} \mathrm{~F}$-choline PET/CT, and WB-MRI $(n=33)$, and the second row represents a comparison between the patients who underwent BS and WB-MRI $(n=56)$

\begin{tabular}{lllc}
\hline & BS & ${ }^{18}$ F-choline PET/CT & WB-MRI \\
\hline N0 & - & $23 / 33(69.7 \%)$ & $26 / 33(78.8 \%)$ \\
& - & - & $43 / 56(76.8 \%)$ \\
N1 & - & $10 / 33(30.3 \%)$ & $7 / 33(21.2 \%)$ \\
& - & - & $13 / 56(23.2 \%)$ \\
M0 & $30 / 33(90.9 \%)$ & $21 / 33(63.6 \%)$ & $22 / 33(66.7 \%)$ \\
& $43 / 56(76.8 \%)$ & - & $34 / 56(60.7 \%)$ \\
M1a & - & $6 / 33(18.2 \%)$ & $3 / 33(9.1 \%)$ \\
& - & - & $6 / 56(10.7 \%)$ \\
M1b & $3 / 33(9.1 \%)$ & $6 / 33(18.2 \%)$ & $8 / 33(24.2 \%)$ \\
& $13 / 56(23.2 \%)$ & - & $16 / 56(28.6 \%)$ \\
M1c & - & $0 / 33(0 \%)$ & $0 / 33(0 \%)$ \\
& - & - & $0 / 56(0 \%)$ \\
\hline
\end{tabular}

at each TNM stage for a cohort of patients undergoing BS, ${ }^{18} \mathrm{~F}$-choline PET/CT, and WB-MRI against the same reference standard (Fig. 2c), using a score of $\geq 4$ as positive for the WB-MRI.

\section{Results}

Fifty-six patients (mean age 67.9 years, range 51.9-84.4 years), median PSA 20.05 (IQR 10.07-61.20). Fifty patients were 'high-risk' and 6 patients 'intermediate-risk'. Maximum Gleason score was $3+3$ for two patients, $3+4$ for nineteen patients, $4+3$ for fourteen patients, $4+4$ for five patients, $4+$ 5 for thirteen patients, and $5+5$ for one patient.

The mean time of radiologists to report each component of the LSR was 15 min for mDixon + DWI, and an additional $6.5 \mathrm{~min}$ for $\mathrm{T} 2 \mathrm{~W}$ and $4 \mathrm{~min}$ for post-contrast scans.

No suspicious lesion (scoring 4, 5, or 6) was identified below the mid-thigh level on any imaging modality. Two cases had suspicious lesions in the cervical and thoracic spine; otherwise, no disease was identified above the diaphragm. The review panel also found that all sites of positive disease on both BS, ${ }^{18} \mathrm{~F}$-choline PET/CT, and WB-MRI were anatomically matched.

The distribution of N/M disease for each imaging modality (BS, ${ }^{18} \mathrm{~F}$-choline PET/CT, and WB-MRI) is presented in Table 2.

Concordance statistics $(\kappa)$ between WB-MRI readers, between WB-MRI consensus and BS, and between WBMRI consensus and ${ }^{18} \mathrm{~F}$-choline PET/CT are presented in Table 3.

ROC-AUC statistics for 'TNM'-based nodal and metastatic status following each part of the LSR are presented in Table 4 against the follow-up based reference standard.

No significant differences were detected between the mean ROC-AUC for each component of the LSR $(p<0.05)$, so the simplest WB-MRI combination was chosen for further analysis (DWI + mDixon). Youden's index confirmed the optimal cutoff of the ROC-AUC was $\geq 4$ in all cases. The sensitivity and specificity for BS, ${ }^{18} \mathrm{~F}$-choline PET/CT, and WB-MRI were therefore calculated using a threshold of $\geq 4$ as positive against the follow-up reference standard. Results are displayed in Table 5, along with their numerators and denominators.

Typical examples of lesions missed on BS which are detected by WB-MRI are provided in Figs. 3 and 4.

\section{Discussion}

WB-MRI is gaining momentum as a staging modality in prostate cancer, but requires further validation prior to being introduced into clinical practice. Firstly, our results show that WBMRI detected more positive bony metastatic (M1b) disease than ${ }^{18} \mathrm{~F}$-choline $\mathrm{PET} / \mathrm{CT}$ and BS with 8,6 , and 3 positive lesions for WB-MRI, ${ }^{18} \mathrm{~F}$-choline $\mathrm{PET} / \mathrm{CT}$, and $\mathrm{BS}$ respectively. We then confirmed that WB-MRI had the highest sensitivity of all modalities for detecting metastatic bone disease: 0.90 vs. 0.80 for ${ }^{18} \mathrm{~F}$-choline PET/CT and 0.60 for BS for specificities of $0.88,0.92$, and 1.00 respectively. This finding is in accordance with a meta-analysis which compared the diagnostic accuracy of BS, ${ }^{18} \mathrm{~F}$-choline PET/CT, and WB-MRI and gave pooled sensitivities and specificities of $0.97 / 0.95,0.91 /$ 0.99, and 0.79/0.82 for WB-MRI, ${ }^{18} \mathrm{~F}$-choline PET/CT, and BS [4] respectively. High diagnostic sensitivity could reflect
Table 3 Interobserver and intermodality concordances

\begin{tabular}{llll}
\hline & $\begin{array}{l}\text { Local nodes } \\
(\mathrm{N} 1)\end{array}$ & $\begin{array}{l}\text { Metastatic nodes } \\
(\mathrm{M} 1 \mathrm{a})\end{array}$ & $\begin{array}{l}\text { Metastatic bones } \\
(\mathrm{M} 1 \mathrm{~b})\end{array}$ \\
\hline $\begin{array}{l}\text { Interobserver concordance for WB-MRI } \\
(n=56)\end{array}$ & 0.79 & 0.68 & 0.58 \\
$\begin{array}{l}\text { Concordance of WB-MRI vs. BS }(n=56) \\
\begin{array}{l}\text { Concordance of WB-MRI vs. }{ }^{18} \text { F-choline } \\
\text { PET/CT }(n=33)\end{array}\end{array}$ & - & - & 0.68 \\
\hline
\end{tabular}

WB-MRI whole-body MRI, $B S^{99 \mathrm{~m}}$ Tc bone scintigraphy 
Table 4 ROC-AUC for each component of the LSR, performed as part of a patientbased analysis according to the reference standard

\begin{tabular}{lllll}
\hline ROC-AUC & N0/N1 $(n=30)$ & M1a $(n=31)$ & M1b $(n=33)$ & Mean ROC-AUC \\
\hline $\begin{array}{l}\text { Reader 1 } \\
\text { mDixon + DWI }\end{array}$ & $0.97(0.91-1.00)$ & $0.99(0.96-1.00)$ & $0.86(0.72-1.00)$ & 0.94 \\
$\quad$ + T2-TSE & $0.98(0.94-1.00)$ & $0.99(0.95-1.00)$ & $0.93(0.84-1.00)$ & 0.96 \\
$\quad$ + contrast & $0.98(0.94-1.00)$ & $0.97(0.91-1.00)$ & $0.90(0.76-1.00)$ & 0.95 \\
Reader 2 & & & & \\
$\quad$ mDixon + DWI & $0.94(0.81-1.00)$ & $0.87(0.60-1.00)$ & $0.86(0.73-1.00)$ & 0.89 \\
$\quad+$ T2-TSE & $0.94(0.82-1.00)$ & $0.87(0.60-1.00)$ & $0.94(0.83-1.00)$ & 0.91 \\
$\quad$ + contrast & $0.94(0.82-1.00)$ & $0.87(0.60-1.00)$ & $0.93(0.82-1.00)$ & 0.91 \\
\hline
\end{tabular}

ROC-AUC receiver operator characteristic area under the curve, LSR locked sequential read, TSE turbo spin echo. Figures in parentheses represent $95 \%$ confidence intervals the fact that DWI sequences are designed to probe small changes in tissue microstructure, as found in the early cellular phase of a metastasis, before a sclerotic reaction has been effected in bone [18].

Table 5 Performance characteristics of BS, ${ }^{18} \mathrm{~F}$-choline PET/CT, and WB-MRI carried out as a patient-based analysis vs. the reference standard

\begin{tabular}{llll}
\hline & N1 $(n=18)$ & M1a $(n=17)$ & M1b $(n=18)$ \\
\hline BS & & & \\
Sensitivity & - & - & $0.60(3 / 5)$ \\
Specificity & & & $1.00(13 / 13)$ \\
PPV & & & $1.00(3 / 3)$ \\
NPV & & & $0.87(13 / 15)$ \\
${ }^{18}$ F-choline PET/CT & & \\
Sensitivity & $1.00(7 / 7)$ & $0.75(3 / 4)$ & $0.80(4 / 5)$ \\
Specificity & $0.82(9 / 11)$ & $0.92(12 / 13)$ & $0.92(12 / 13)$ \\
PPV & $0.77(7 / 9)$ & $0.75(3 / 4)$ & $0.80(4 / 5)$ \\
NPV & $1.00(9 / 9)$ & $0.92(12 / 13)$ & $0.92(12 / 13)$ \\
WB-MRI: reader 1 & & & $0.80(4 / 5)$ \\
Sensitivity & $1.00(7 / 7)$ & $1.00(4 / 4)$ & $1.00(13 / 13)$ \\
Specificity & $0.91(10 / 11)$ & $0.85(11 / 13)$ & $1.00(4 / 4)$ \\
PPV & $0.88(7 / 8)$ & $0.67(4 / 6)$ & $0.93(13 / 14)$ \\
NPV & $1.00(10 / 10)$ & $1.00(11 / 11)$ & \\
WB-MRI: reader 2 & & & $0.00(5 / 5)$ \\
Sensitivity & $1.00(7 / 7)$ & $0.50(2 / 4)$ & $0.76(10 / 13)$ \\
Specificity & $1.00(11 / 11)$ & $1.00(13 / 13)$ & $0.62(5 / 8)$ \\
PPV & $1.00(7 / 7)$ & $1.00(2 / 2)$ & $1.00(10 / 10)$ \\
NPV & $1.00(11 / 11)$ & $0.86(13 / 2)$ & \\
WB-MRI mean & & & 0.97 \\
Sensitivity & 1.00 & 0.93 & \\
Specificity & 0.96 & 0.83 & \\
PPV & 0.94 & 0.93 & \\
NPV & 1.00 & & \\
\hline
\end{tabular}

$W B-M R I$ whole-body MRI, $B S^{99 \mathrm{~m}} \mathrm{Tc}$ bone scintigraphy, $N P V$ negative predictive value, $P P V$ positive predictive value. WB-MRI mean is the mean of the two readers
High and very similar sensitivities/specificities were also shown for WB-MRI and ${ }^{18} \mathrm{~F}$-choline PET/CT for nodal disease, with values of 1.00/0.96 and 1.00/0.82 for N1 disease and $0.75 /$ 0.93 and $0.75 / 0.92$ for M1a disease respectively. Both of the cross-sectional modalities therefore appear more accurate than conventional CT, which is again in accordance with a metaanalysis which reported pooled sensitivities and specificities of $0.42 / 0.82$ for CT [3], vs. 0.49/0.95 for choline PET/CT [5]. MRI studies which incorporate DWI into their scanning protocols report a heterogenous sensitivity for lymph nodes which ranges from 0.17 [19] to 0.73 [20]. Whilst both of these studies used extended pelvic lymph node dissection as the reference standard, the lower sensitivity reported by Pinaquy and colleagues [19] could relate to their chosen $b$-values of 0 and $100 \mathrm{~s} / \mathrm{mm}^{2}$, which contravenes the recommendations of international consensus guidelines [21], and emphasises the need for optimised scanning technique. In concordance with the findings of our study, the specificity of MRI for nodal detection is thought to be high, with a limited number of studies quoting values ranging from $86 \%$ [20] to $98 \%$ [22].

A potential strength of our study was the use of a vertex-tofeet protocol which enabled direct comparison with BS and could assess potential lesions outside of the field of view for ${ }^{18} \mathrm{~F}$-choline PET/CT. Whole-body cross-sectional studies regarding disease distribution in the PSA screening era are welcome since strongest data regarding disease distribution is provided by an autopsy study prior to PSA screening era, which did not routinely examine the peripheral skeleton [23]. Complete body coverage has been both suggested $[24,25]$ and deemed unnecessary [9], which is perhaps could partially be due to the uncertainty regarding disease distribution in the PSA screening era. Since no lesions were detected below the knee or extravertebral lesions above the diaphragm, our data suggests that scanning below the knee may indeed be unnecessary, and a cervical and thoracic spine MRI may be a reasonable compromise for detecting disease above the diaphragm, and is in keeping with the findings of another study [9], which reported all patients with peripheral metastases occurring in high-risk prostate cancer $(60$ in total) also had vertebral metastases, and no metastases 



Fig. 3 Example of discordant skeletal site in a 64-year-old man with a serum prostate-specific antigen level of 25.2. Top left: Negative ${ }^{99 \mathrm{~m}} \mathrm{Tc}$ bone scintigram (BS), Top right: ${ }^{18} \mathrm{~F}$-choline PET/CT showing an area of increased tracer avidity at the right inferior pubic ramus consistent with a metastasis. Bottom left: Axial T2W TSE showing the lesion is of low

occurred below the knee. With further confirmatory work, scanning the abdomen, pelvis, and femora using pre-contrast mDixon and DWI at $2 b$-values paired with a whole spine MRI as a routine staging examination could be applied and would have approximately 700 images, vs. 12,000 images per patient in the present study. Reducing the number of images may further improve the interobserver concordance by reducing the complexity of imaging datasets. We found interobserver concordance to be 'substantial' for N1 and M1a disease ( $\kappa=0.79$ and 0.68 respectively), and 'moderate' for M1b (0.58), whereby the lower concordance in bone metastases could be explained by the nonspecific features of bone lesions on MRI, and the fact that acquisitions were tailored for WB cancer staging rather than bone lesion characterisation. Furthermore, the more subjective criteria applied for assessing bone lesions vs. nodal size measurements may have given rise to further heterogeneity in the data and thus lower levels of concordance.

The LSR paradigm allowed the incremental value of additional sequences to be assessed, whereby adding $\mathrm{T} 2 \mathrm{~W}$ and postcontrast mDixon sequences did not improve ROC-AUC significantly. These results could be used to streamline WB-MRI scanning protocols in research and clinical practice. For example, performing pre-contrast mDixon + DWI alone could save 10-min reporting time and 20-min scan time and avoid the need for cannulation and gadolinium administration. Furthermore, as suggested by the MET-RADS-P consensus guidelines [25], the use of $2 b$-values rather than 4 could be sufficient — especially for primary staging purposes, which would reduce scan time by a further $25 \mathrm{~min}$. Whilst the MET-RADS-P guidelines were based on expert opinion, WB-Dixon and DWI were recommended in
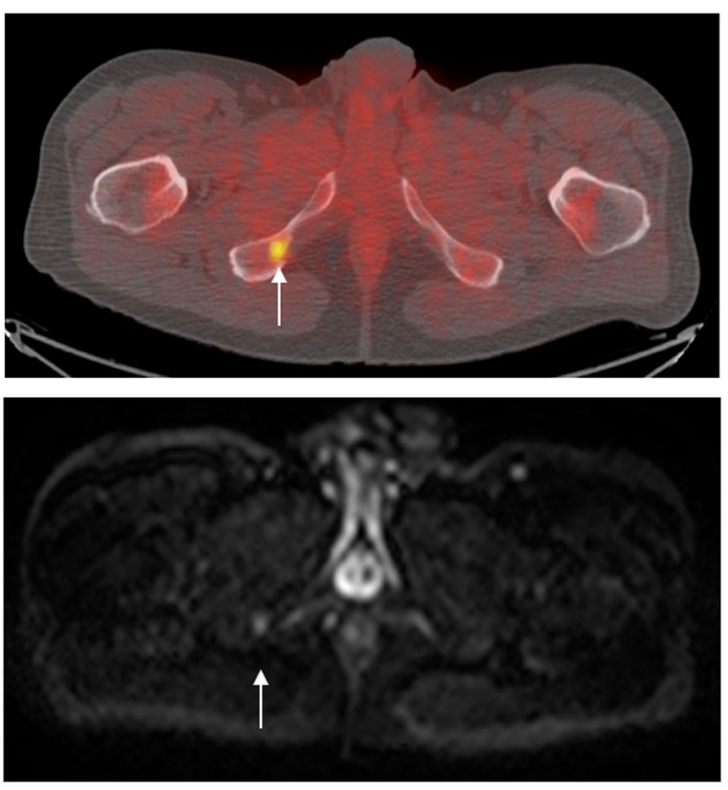

signal intensity. Bottom right: the lesion has increased diffusion-weighted signal on $b=1000 \mathrm{~s} / \mathrm{mm}^{2}$. The lesion was considered as an example of a true positive whole-body MRI, true positive ${ }^{18} \mathrm{~F}$-choline PET/CT, and false negative $\mathrm{BS}$

combination with whole spine $\mathrm{T} 1$ and short tau inversion recovery (STIR), meaning our findings provide evidence to support a similar simple scanning protocol when characterising oncological burden in prostate cancer.

Another potential strength of our study was the choice of a reference standard based on follow-up MRI rather than on best value comparator (BVC) alternatives [8] which rely upon imaging tests such as BS and plain radiographs with limited performance characteristics. Whilst TP was assigned without follow-up imaging when BS and MRI were concordant due to the high specificity of BS in the context of prostate cancer, we did not assign TN without MRI follow-up, since genuine lack of sensitivity, i.e. FN results on both modalities, is also possible.

The limitations of this study include patient number, its singlecentre nature, and a relatively low number of positive cases. Furthermore, not all patients underwent ${ }^{18} \mathrm{~F}$-choline PET/CT, meaning patients with a negative $\mathrm{BS}$ in whom the suspicion for metastatic disease remained high may have been more likely to be selected for ${ }^{18} \mathrm{~F}$-choline PET/CT vs. patients with clear evidence of metastases on BS, leading to lower apparent levels of diagnostic accuracy for ${ }^{18} \mathrm{~F}$-choline PET/CT.

In addition, not all patients underwent WB-MRI follow-up at 1 year, which could lead to selection bias, e.g. patients who were feeling well or unwell at the time of follow-up may be more likely to refuse the second scan. However, the most common reason provided at telephone consultation was that they had incurred too many imaging tests and therefore declined further participation. Whilst incorporation bias likely gave rise to the high values of sensitivity and specificity (e.g. vs. pelvic lymph node dissection (PLND) as a nodal reference standard), it would 

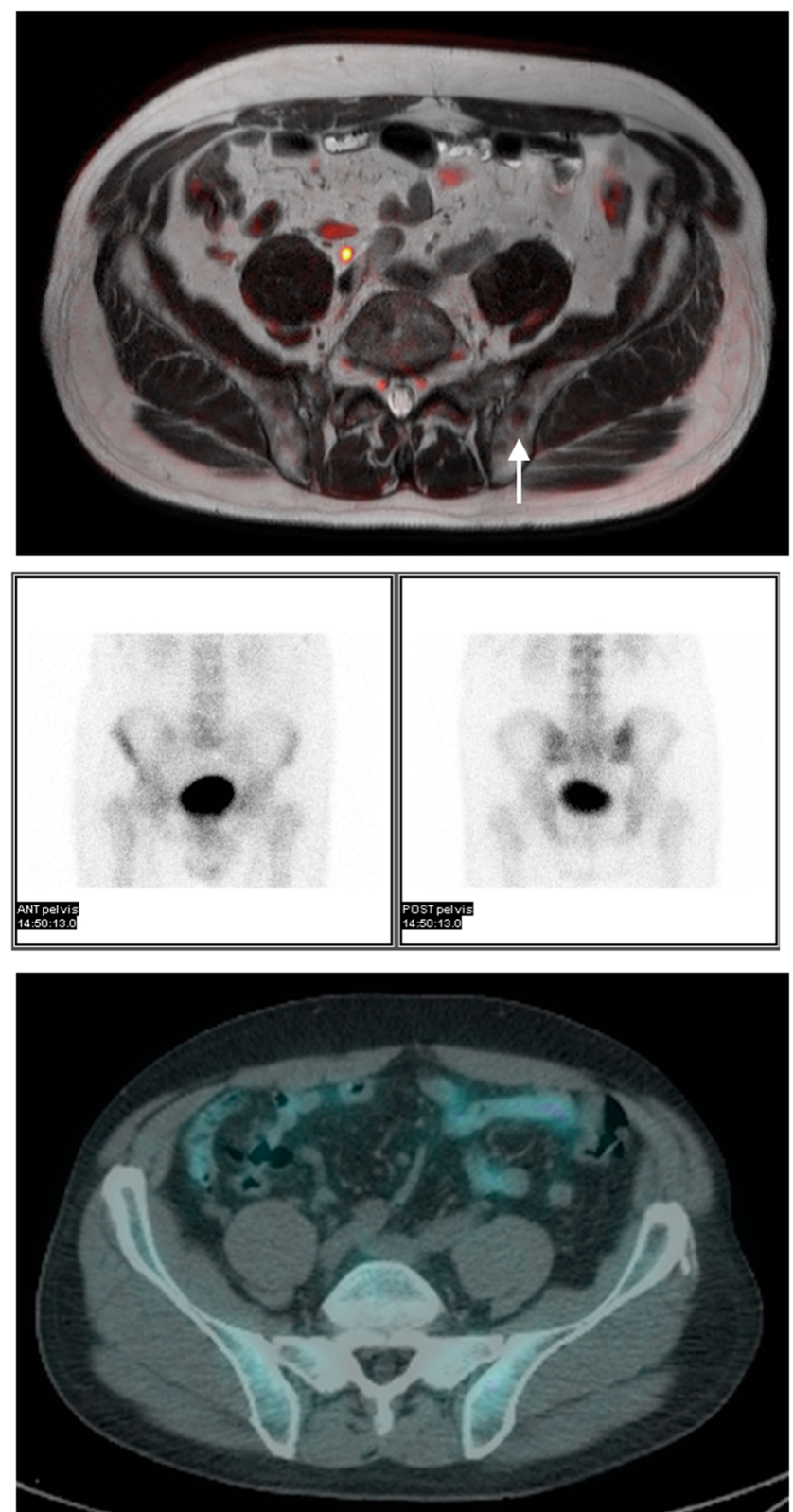

Fig. 4 Example of a discordant skeletal site in a 73-year-old man with a serum prostate-specific antigen level of 19.4. Top: Axial T2-weighted turbo spin echo with fused $b=1000 \mathrm{~s} / \mathrm{mm}^{2}$ showing a metastasis in the left iliac body, which is occult on BS (middle) and ${ }^{18} \mathrm{~F}$-choline PET/CT (bottom). The site was considered to represent a true positive whole-body MRI, false negative ${ }^{18} \mathrm{~F}$-choline PET/CT, and false negative ${ }^{99 \mathrm{~m}} \mathrm{Tc}$ bone scintigram

not have been practical or ethically acceptable to perform nodal dissection for the purposes of the study, and selecting patients who are undergoing PLND would incur spectrum bias.

Further work could include performing a lesion-based analysis in the same cohort of patients, whereby the number of lesions detected and their anatomical sites could be established. Further validation of WB-MRI could also be carried out in multicentre trials, where economic and clinical utility could also be considered. The findings of the present study are not limited to WB-MRI and can be used to inform rational PET-MRI protocols, e.g. in combination with prostate-specific membrane antigen (PSMA) PET tracer.

\section{Conclusion}

WB-MRI provides high levels of diagnostic accuracy for both nodal and metastatic bone disease, with higher levels of sensitivity than BS for metastatic disease, and similar performance to ${ }^{18} \mathrm{~F}$-choline PET/CT. T2 and post-contrast mDixon had no significant additive value above a protocol comprising mDixon and DWI alone.

Acknowledgements We would like to thank Dr Reena Davda for her help reviewing patients according to the reference standard.

Funding This work was undertaken at UCLH/UCL, which received a proportion of its funding from the NIHR Biomedical Research Centres funding scheme of the UK Department of Health. The work of AL, HSS, and SP is supported by the KCL/UCL Comprehensive Cancer Imaging Centre.

\section{Compliance with ethical standards}

Guarantor The scientific guarantor of this publication is Shonit Punwani.

Conflict of interest The authors declare that they have no conflict of interest.

Statistics and biometry No complex statistical methods were necessary for this paper.

Informed consent Written informed consent was obtained from all subjects (patients) in this study.

Ethical approval Institutional Review Board approval was obtained.

Methodology

- Prospective diagnostic study performed at one institution

Open Access This article is distributed under the terms of the Creative Commons Attribution 4.0 International License (http:// creativecommons.org/licenses/by/4.0/), which permits unrestricted use, distribution, and reproduction in any medium, provided you give appropriate credit to the original author(s) and the source, provide a link to the Creative Commons license, and indicate if changes were made.

\section{References}

1. Buyyounouski MK, Choyke PL, McKenney JK et al (2017) Prostate cancer - major changes in the American Joint Committee on Cancer eighth edition cancer staging manual. CA Cancer J Clin 67:245-253. https://doi.org/10.3322/caac.21391 
2. Bjurlin MA, Rosenkrantz AB, Beltran LS, Raad RA, Taneja SS (2015) Imaging and evaluation of patients with high-risk prostate cancer. Nat Rev Urol 12:617-628. https://doi.org/10.1038/nrurol. 2015.242

3. Hövels AM, Heesakkers RA, Adang EM et al (2008) The diagnostic accuracy of CT and MRI in the staging of pelvic lymph nodes in patients with prostate cancer: a meta-analysis. Clin Radiol 63:387-395

4. Shen G, Deng H, Hu S, Jia Z (2014) Comparison of choline-PET/ CT, MRI, SPECT, and bone scintigraphy in the diagnosis of bone metastases in patients with prostate cancer: a meta-analysis. Skeletal Radiol 43:1503-1513

5. Evangelista L, Guttilla A, Zattoni F, Muzzio PC, Zattoni F (2013) Utility of choline positron emission tomography/computed tomography for lymph node involvement identification in intermediate- to high-risk prostate cancer: a systematic literature review and metaanalysis. Eur Urol 63:1040-1048

6. Kapoor V, McCook BM, Torok FS (2004) An introduction to PETCT imaging. RadioGraphics 24:523-543. https://doi.org/10.1148/ rg. 242025724

7. Linton KD, Catto JW (2012) Whole-body magnetic resonance imaging and prostate cancer metastases: a new gold standard of detection, but does it help us and at what cost? Eur Urol 62:76-77

8. Lecouvet FE, El Mouedden J, Collette L et al (2012) Can wholebody magnetic resonance imaging with diffusion-weighted imaging replace tc $99 \mathrm{~m}$ bone scanning and computed tomography for single-step detection of metastases in patients with high-risk prostate cancer? Eur Urol 62:68-75

9. Lecouvet FE, Simon M, Tombal B, Jamart J, Vande Berg BC, Simoni P (2010) Whole-body MRI (WB-MRI) versus axial skeleton MRI (AS-MRI) to detect and measure bone metastases in prostate cancer (PCa). Eur Radiol 20:2973-2982

10. Mosavi F, Johansson S, Sandberg DT, Turesson I, Sörensen J, Ahlström H (2012) Whole-body diffusion-weighted MRI compared with $18 \mathrm{~F}-\mathrm{NaF}$ PET/CT for detection of bone metastases in patients with high-risk prostate carcinoma. AJR Am J Roentgenol 199:1114-1120

11. Venkitaraman R, Cook GJ, Dearnaley DP et al (2009) Whole-body magnetic resonance imaging in the detection of skeletal metastases in patients with prostate cancer. J Med Imaging Radiat Oncol 53: 241-247

12. Pasoglou V, Larbi A, Collette L et al (2014) One-step TNM staging of high-risk prostate cancer using magnetic resonance imaging (MRI): toward an upfront simplified "all-in-one" imaging approach? Prostate 74:469-477

13. Perera M, Papa N, Christidis D et al (2016) Sensitivity, specificity, and predictors of positive $68 \mathrm{Ga}$-prostate-specific membrane antigen positron emission tomography in advanced prostate cancer: a systematic review and meta-analysis. Eur Urol 70:926-937

14. Boorjian SA, Karnes RJ, Rangel LJ, Bergstralh EJ, Blute ML (2008) Mayo Clinic validation of the D'Amico risk group classification for predicting survival following radical prostatectomy. J Urol 179:1354-1361

15. Landis JR, Koch GG (1977) The measurement of observer agreement for categorical data. Biometrics 33:159-174

16. Hanley JA, McNeil BJ (1982) The meaning and use of the area under a receiver operating characteristic (ROC) curve. Radiology 143:29-36

17. Youden WJ (1950) Index for rating diagnostic tests. Cancer 3:3235

18. Padhani AR, Gogbashian A (2011) Bony metastases: assessing response to therapy with whole-body diffusion MRI. Cancer Imaging 11(Spec No A):S129-S145. https://doi.org/10.1102/ 1470-7330.2011.9034

19. Pinaquy JB, De Clermont-Galleran H, Pasticier G et al (2015) Comparative effectiveness of [(18)F]-fluorocholine PET-CT and pelvic MRI with diffusion-weighted imaging for staging in patients with high-risk prostate cancer. Prostate 75:323-331

20. Thoeny HC, Froehlich JM, Triantafyllou M et al (2014) Metastases in normal-sized pelvic lymph nodes: detection with diffusionweighted MR imaging. Radiology 273:125-135

21. Padhani AR, Liu G, Koh DM et al (2009) Diffusion-weighted magnetic resonance imaging as a cancer biomarker: consensus and recommendations. Neoplasia 11:102-125

22. Van den Bergh L, Lerut E, Haustermans K et al (2015) Final analysis of a prospective trial on functional imaging for nodal staging in patients with prostate cancer at high risk for lymph node involvement. Urol Oncol 33:109.e23-109.e31

23. Bubendorf L, Schöpfer A, Wagner U et al (2000) Metastatic patterns of prostate cancer: an autopsy study of 1,589 patients. Hum Pathol 31:578-583

24. Haubold-Reuter BG1, Duewell S, Schilcher BR, Marincek B, von Schulthess GK (1993) The value of bone scintigraphy, bone marrow scintigraphy and fast spinecho magneticresonance imaging in staging of patients with malignant solid tumours: a prospectivestudy. Eur J Nucl Med 20(11):1063-9

25. Padhani AR, Lecouvet FE, Tunariu N, Koh DM, De Keyzer F, Collins DJ, Sala E, Schlemmer HP, Petralia G, Vargas HA, Fanti S, Tombal HB, de Bono J (2017) METastasis Reporting and Data System for Prostate Cancer: Practical Guidelines for Acquisition, Interpretation, and Reporting of Whole-body Magnetic Resonance Imaging-based Evaluations of Multiorgan Involvement in Advanced Prostate Cancer. Eur Urol 71(1):81-92. https://doi.org/ 10.1016/j.eururo.2016.05.033 